\title{
Measuring fidelity of delivery of the Community Occupational Therapy in Dementia-UK intervention
}

\author{
Holly Walton ${ }^{1 *}$ (D, Ildiko Tombor ${ }^{2}$, Jane Burgess ${ }^{3}$, Hilary Groarke ${ }^{4}$, Tom Swinson ${ }^{5}$, Jennifer Wenborn ${ }^{6,3}$, \\ Aimee Spector ${ }^{4}$, Martin Orrell, Gail Mountain ${ }^{8}$ and Susan Michie ${ }^{4}$
}

\begin{abstract}
Background: Interpreting data about intervention effectiveness requires an understanding of which intervention components were delivered and whether they were delivered as planned (fidelity of delivery). These studies aimed to develop a reliable measure for assessing fidelity of delivery of the Community Occupational Therapy in Dementia-UK intervention (COTiD-UK) (Study 1) and measure fidelity of delivery of COTiD-UK across sessions, sites and occupational therapists (Study 2).

Methods: The studies used a longitudinal observational design nested within a multi-site randomised controlled trial. Where practicable, all intervention sessions were audio-recorded. Fidelity checklists and coding guidelines were developed, piloted and refined until good agreement was achieved between two coders. Ten percent of sessions were purposively sampled from 12 sites and 31 occupational therapists. Transcripts were coded using checklists developed in Study 1; 10\% of sets of intervention session transcripts were double coded to ensure that agreement was maintained. Percentages of components that were delivered were calculated for each session, site and occupational therapist.

Results: A reliable measure of fidelity of delivery for COTiD-UK was developed after several rounds of piloting and amendments. COTiD-UK was delivered with moderate fidelity across all six sessions (range: 52.4-75.5\%). The mean range of fidelity varied across sites (26.7-91.2\%) and occupational therapists (26.7-94.1\%).

Conclusions: A reliable, systematic method for measuring fidelity of delivery of COTiD-UK was developed and applied, and can be adapted for use in similar interventions. As COTID-UK was delivered with moderate fidelity, there is a reasonable degree of confidence that intervention effects were attributable to COTiD-UK.
\end{abstract}

Keywords: Fidelity of delivery, Implementation, Dementia, Occupational therapy, Complex intervention

\section{Background}

Dementia is a global health concern, with 115.4 million people expected to receive a diagnosis of dementia by 2050 [1]. Psychosocial interventions are developed to support people with dementia and their family carers in maintaining their quality of life. Interpreting the effectiveness of psychosocial interventions requires knowledge of the extent to which the intervention was delivered as planned (termed 'fidelity of delivery') [2-4]. Whilst

\footnotetext{
* Correspondence: holly.walton@ucl.ac.uk

${ }^{1}$ Department of Applied Health Research, University College London, 1-19

Torrington Place, London, UK

Full list of author information is available at the end of the article
}

psychosocial interventions for people with dementia may show promise, it is not always clear whether they have been delivered as planned [5].

One such intervention is the Community Occupational Therapy in Dementia (COTiD) intervention, which was first developed and delivered in the Netherlands [6, 7]. COTiD aimed to facilitate independence, meaningful activity and quality of life among people with mild to moderate dementia and their families [6]. COTiD was delivered to people with dementia and their family carers over 10 one hour sessions over five weeks [6]. The first four sessions focused on people with dementia and family carers choosing and prioritising meaningful activities that

(c) The Author(s). 2019 Open Access This article is distributed under the terms of the Creative Commons Attribution 4.0 International License (http://creativecommons.org/licenses/by/4.0/), which permits unrestricted use, distribution, and reproduction in any medium, provided you give appropriate credit to the original author(s) and the source, provide a link to the Creative Commons license, and indicate if changes were made. The Creative Commons Public Domain Dedication waiver (http://creativecommons.org/publicdomain/zero/1.0/) applies to the data made available in this article, unless otherwise stated. 
they wanted to improve, by interviewing both the person with dementia and family carer [6]. Adaptations to the home and environment were considered as part of these sessions. In the final six sessions, problem solving skills and coping strategies were developed to enhance the use of daily activities [6]. A single site randomised controlled trial (RCT) of COTiD found that both people with dementia and their family carers benefitted from the intervention [6, 7]. For example, people with dementia's daily functioning and family carers' sense of competence increased. Both groups experienced better quality of life than those who did not receive the intervention. However, when the intervention was replicated in Germany, no significant differences between intervention and control groups were found in a seven-site RCT $[8,9]$.

Differences in the effectiveness of COTiD could be attributed to: differing cultural contexts, translation of the intervention, differences in measures used, or differences in control groups. For example, in the German study, participants in the control group received a leaflet and one consultation visit from an occupational therapist [8], whereas participants in the control group in the Netherlands did not receive any occupational therapy [6]. It is also possible that differences could have been a result of how the intervention was delivered. Previous research has found that complex healthcare interventions are often not delivered as planned [10, 11]. In Germany, self-reported fidelity of delivery was reported to be $78 \%$ in the COTiD intervention group and $80 \%$ in the control group. A few intervention components were poorly delivered, suggesting that at least some aspects of COTiD had poor fidelity of delivery [8]. Previous studies of the COTiD intervention did not use the same measure of fidelity or ensure their reliability; therefore, fidelity of delivery cannot be accurately compared between Germany and the Netherlands.

To understand whether it is possible to deliver an intervention as planned and whether variable intervention effects could be the result of differences in intervention fidelity, it is important to measure fidelity. The current gold-standard is to record all sessions and select a representative sample to transcribe and compare against an intervention-specific checklist of intervention components $[2,3,10,12,13]$. To ensure that measurements are reliable, it is recommended that intervention transcripts are coded by multiple researchers [14]. To determine the trustworthiness of fidelity assessments, the psychometric quality of measures (e.g. reliability and validity) should be considered when developing and reporting them [15]. Fidelity should be monitored across providers, sites and participants to account for differences in context $[10,16]$.

The COTiD intervention was translated and adapted for the UK context [Wenborn et al., in preparation]. The Community Occupational Therapy in Dementia UK
(COTiD-UK) intervention involves home and communitybased occupational therapy with people with mild to moderate dementia and a family carer (referred to as 'dyads'). COTiD-UK is designed to be delivered by occupational therapists. COTiD-UK was evaluated in a multi-centre, pragmatic, single blind RCT (COTiD-UK vs. treatment as usual) between October 2014 and July 2017. An embedded qualitative study involved qualitative interviews with occupational therapists and dyads to explore the experience of participating in the COTiD-UK intervention (for more information about COTiD-UK and the RCT, see [17]). Four hundred and sixty-eight dyads were recruited and randomly allocated to either the COTiD-UK intervention $(n=249)$ or a treatment as usual control group $(n=219)$.

Dyads in the intervention group received up to $10 \mathrm{~h}$ of COTiD-UK over approximately 10 weeks. Seven key sessions were delivered: 1 . Introduction (occupational therapist introduced self and the COTiD-UK format), 2. Occupational Performance History Interview (OPHI) [18] (occupational therapist interviewed the person with dementia about their life, experiences and activities), 3. Ethnographic Interview (occupational therapist interviewed the family carer about their life and experience of providing care), 4. Summaries of interviews and observations (occupational therapist summarised the information gathered from the interviews, together with their own observations made within the context of an environmental and activity assessment), 5. Goal-setting (occupational therapist facilitated the dyad to create Specific, Measurable, Achievable, Realistic and Timed (SMART) goals), 6. Consultation and advice (occupational therapist enabled the carer to develop problem solving skills and provided other relevant advice and information), and 7. Evaluation (occupational therapist, person with dementia and family carer reviewed their progress in achieving the set goals). These six or seven sessions (depending on whether Summaries and Goal-setting were delivered separately or together) were collectively referred to as a 'set'.

All occupational therapists received two days of faceto-face training in how to deliver the COTiD-UK intervention. They initially delivered the intervention to a 'training dyad', audio-recording all sessions as practicable. A COTiD-UK trainer listened and assessed the training dyad recordings to determine if the occupational therapist had achieved the necessary competencies to deliver COTiD-UK within the RCT. To enable occupational therapists to consolidate their COTiDUK understanding, knowledge and skills and give opportunity for reflection, a follow-up training day was provided once they had delivered COTiD-UK. All occupational therapists participated in supervision throughout the delivery of COTiD-UK. Supervision models varied between peer, group and individual supervision, depending upon the availability of a COTiD-UK supervisor. 
To understand the effectiveness of COTiD-UK, it was necessary to measure fidelity alongside the delivery of the trial. This research therefore aimed to: develop a measure for assessing fidelity of delivery of COTiD-UK (Study 1), and measure fidelity of delivery of COTiD-UK across intervention sessions, sites and occupational therapists (Study 2).

\section{Ethical approval (both studies)}

All ethical and research governance requirements were followed. Ethical approval was obtained from: NRES Committee London - Camberwell St Giles, REC Reference number: 14/LO/0736. Encrypted audio-recorders were used and data transferred using encrypted memory sticks. Data were transcribed by a professional transcription company. All transcripts were fully anonymised and thus individuals were unidentifiable from the data or resulting outputs.

Study 1: The development of a measure to assess fidelity of delivery of COTiD-UK.

\section{Methods}

Design (both studies)

A longitudinal observational study nested within the COTiD-UK RCT was conducted across 12 of the 15 National Health Service (NHS) trust research sites that were involved in the trial, in England. COTiD-UK was delivered primarily by occupational therapists recruited from clinical services within participating organisations but in some cases was also delivered by research staff who were also occupational therapists. In these cases, occupational therapist researchers did not collect research data from their own participants.

\section{Procedure}

\section{Development of checklists}

The development of fidelity of delivery checklists was informed by the methodology used to assess the fidelity of another psychosocial intervention for people living with dementia: Promoting Independence in Dementia (PRIDE) [19]. A team of behavioural scientists and occupational therapy research staff were involved in the development of the checklists.

The first step was to develop an intervention framework by identifying key components of the intervention. The COTiD-UK checklist template, the COTiD-UK training materials, and discussion with the COTiD-UK trainers informed this step. The COTiD-UK checklist was used by the occupational therapists to record the intervention provided in each session to each dyad as follows: 1) the date and length, 2) who was present, 3) location, 4) COTiD-UK components delivered, 5) travel time, 6) preparation time and 7) clinical recording time. Identifying key components involved going through each of the intervention materials and identifying active ingredients that occupational therapists needed to deliver in each session as part of the COTiD-UK intervention.

The resulting intervention framework outlined: 1) key targets, 2) key components (these were subsequently referred to as 'appointment activities' in the fidelity checklists), 3) the session(s) the component should be delivered in, and 4) whether the component is aimed at the person with dementia or the family carer or both (see Additional file 1).

The intervention framework was used to develop a fidelity checklist for each of the seven key intervention sessions. The 'Summaries' and 'Goal-setting' sessions were combined as they were often delivered together, resulting in six sessions for coding. The fidelity checklists included information on: 1) set identification number, 2) component number, 3) date of the session and 4) key components for each session. Key components were listed as 'occupational therapist behaviours' and components were recorded as 'done', 'done to some extent', 'not done' or 'delivered in a different session'. To check for accuracy of content and comprehensibility of the components in the checklists, feedback on the intervention framework and checklists was then sought from the researchers and occupational therapy research staff involved in the fidelity assessments and training and delivery of COTiD-UK. To ensure that components accurately represented the intervention, components were discussed with the wider team which included occupational therapy research staff who were involved in the training of COTiD-UK providers. This feedback identified that key occupational therapy skills were missing from the framework (e.g. activity analysis, selection, adaptation and grading). To address this, we reviewed relevant occupational therapy literature [20] to identify and operationalise these skills for inclusion in the framework.

Coding guidelines for the fidelity assessment (see Additional file 2) were developed. The coding guidelines included: 1) instructions on how to code transcripts, 2) definitions for each component within each of the intervention sessions and 3) criteria for 'done', 'done to some extent' and 'not done' rating. Examples were also given to illustrate what should be coded as 'done', 'done to some extent', 'not done'. Self-reported goal-setting forms (completed by the occupational therapists), the COTiD-UK leaflet and OPHI and Ethnographic interview questions were used to inform the coding guidelines and to facilitate decision making about what counted as 'done', 'done to some extent' and 'not done'. For example: the topics listed within the OPHI and Ethnographic interview questions were used to distinguish between 'done' (open questions about 5+ topics), 'done to some extent' (open questions about 2-4 topics) or 'not done' (open questions about $0 / 1$ topics). 


\section{Piloted and refined checklists and coding guidelines to improve reliability}

Fidelity checklists and coding guidelines were then piloted and inter-rater agreement was calculated. Piloting initially involved two sets of sessions which were independently coded by three coding pairs. Three coders were naïve to the intervention and three coders had knowledge of it [21]. Disagreements and inter-rater agreement for each coding pair were calculated. If high levels of agreement were not achieved on the first set, the first author met with the coders to discuss disagreements and amend the coding guidelines accordingly before the second set of transcripts was coded. After all pairs completed the coding for these two sets, components that had poor reliability were identified and coding guidelines were amended.

After initial piloting, nine further sets were coded and the initial two sets were re-coded by one and/or two coding pairs (1st, 3rd, 4th, 5th authors). Discrepancies were discussed and coding guidelines and checklists were amended until good agreement was achieved.

The checklists and coding guidelines went through many amendments throughout various stages of the checklist development before being finalised. Changes were made to provide clarity and attempt to increase reliability. There were too many changes to provide a complete list, but some examples are given below. Some of the components relating to style of communication were difficult to code and amendments were made to operationalise these guidelines further. For example, the guidelines for 'used open questions' were changed from 'asking open questions when appropriate' to specifying how many topics would need to be discussed using open questions to count as 'done', 'done to some extent' and 'not done'. Other components were difficult to code because of the way components were worded. Amendments were made to address these difficulties. For example, we merged three goal-setting components (setting goals for the person with dementia, family carer and both) to create two components that were more reliable to code (set at least one individual or joint goal for the person with dementia and family carer and developed these goals into SMART goals). Similarly, for other components we added extra information into the coding guidelines to reduce uncertainty and subjectivity for coders.

\section{Analysis}

Inter-rater agreement was calculated using the Kappa statistic ( $\mathrm{K}$ ) [22] and percentage agreement. To account for the ordinal nature of responses, weighted Kappa was used. This meant that 'partial agreements' were considered [23]. For example: some combinations of responses e.g. 'done' and 'done to some extent' are closer in agreement than others, e.g. 'done' and 'not done'. A threshold of $>0.61 \mathrm{Kappa}$, established for three consecutive sets, was chosen after initial coding demonstrated that $>0.8$ Kappa would be too difficult to achieve consistently. The $>0.61$ Kappa threshold is considered 'good' agreement [24]. Kappa provides a conservative estimate of reliability [21]. Therefore, by lowering the threshold it was still possible to achieve good agreement whilst also accounting for chance agreement. Adjustments were made to the checklists and coding guidelines until good levels of inter-rater reliability were obtained.

\section{Results}

\section{Development of checklists}

Six checklists (from seven sessions) were developed, each containing standardised intervention components (Introduction: 15 components, OPHI: 16 components, Ethnographic interview: 16 components, Summary and Goal-setting: 17 components, Consultation and advice: 15 components, Evaluation: eight components). See Fig. 1 for an example of the checklists. See Additional file 3 for all six checklists.

\section{Piloted and refined checklists and coding guidelines to improve reliability}

Table 1 outlines the inter-rater agreement (weighted Kappa and percentage agreement) achieved for each session in the piloting phase. Good inter-rater agreement $(\kappa>.61)$ was achieved after coding six Introduction transcripts $(\kappa=0.7)$, six OPHI transcripts $(\kappa=0.8-0.9)$, ten Ethnographic transcripts $(\kappa=0.7-0.8), 13$ Summary and Goal-setting transcripts $(\kappa=0.8-0.9)$, eight Consultation and advice transcripts $(\kappa=0.6-0.9)$ and 11 Evaluation transcripts $(\kappa=0.7-0.8)$. For the Summary and Goalsetting session, >.61 Kappa was achieved three times in the last five sets $(\kappa=0.6-0.9)$. However, for this session good agreement (>.61 Kappa) was not achieved three times in a row due to an unequal distribution of responses, i.e. a large number of the same responses [25], which meant that weighted Kappa was moderate $(\kappa=$ 0.4 ) but percentage agreement was very high (82.4\%).

Study 2: Measuring fidelity of delivery of COTiD-UK across sessions, sites and occupational therapists.

\section{Methods \\ Sampling}

Figure 2 illustrates the sampling strategy. A sample of $10 \%$ of audio-recorded intervention sessions were purposively selected, transcribed and analysed, giving 24 sets. As each set included six or seven COTiD-UK sessions, depending on whether 'Summary and Goal-setting' sessions were delivered separately or together, the total number of sessions transcribed potentially ranged from 144 to 168 . In cases where 'Summary and Goal-setting' were delivered separately, these are referred to in the results as Goal-setting 1 (Summary) and 2 (Goal-setting). These sets were purposively sampled 

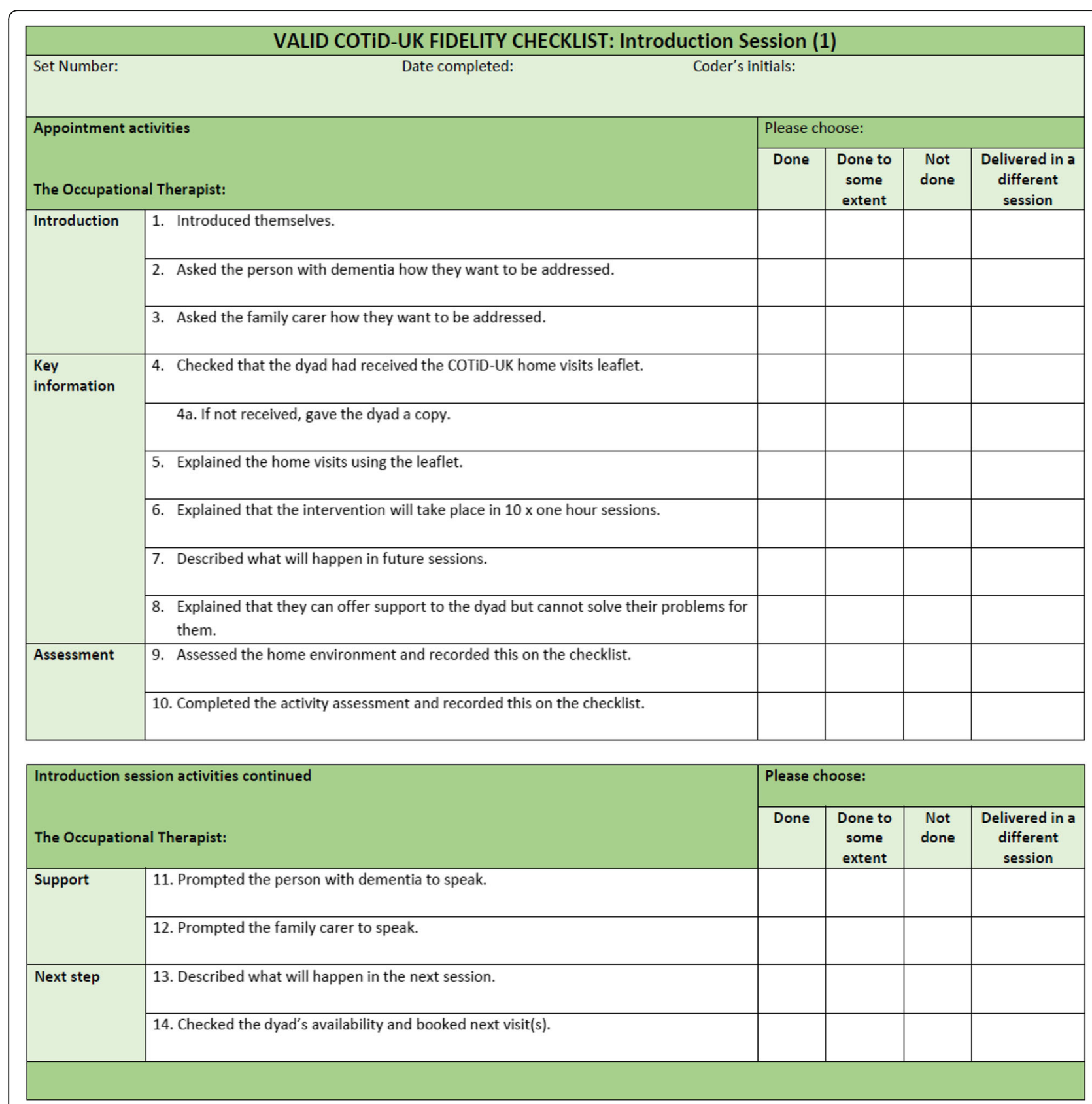

Fig. 1 COTiD-UK Introduction (1) checklist

from 12 of the 15 trial sites and 28 of the 31 occupational therapists. One of the 15 field work sites did not recruit any dyads, another was unable to provide the intervention and one did not have sufficient recordings for sampling; therefore they were omitted. Two sets were selected from each of the remaining 12 sites. Purposive sampling was used to ensure that we sampled from a range of occupational therapists from different sites, and to ensure that the transcripts selected were complete sets. To take occupational therapists' prior clinical and specific COTiD-UK experience into account, recordings were sampled from different therapists and from dyads that were recruited early in the intervention period (e.g. an occupational therapist's second dyad) and dyads that were recruited near the end of the intervention period (e.g. an occupational therapist's ninth dyad). If no full sets were available $(n=8)$, sets which had the majority of sessions were sampled.

\section{Materials}

The checklists developed in Study 1 were used to measure fidelity across sessions, sites and occupational therapists in Study 2. See Study 1 for more details. 
Table 1 Weighted Kappa and percentage agreement scores for each session by round of pilot coding, set of transcripts and coding pair

\begin{tabular}{|c|c|c|c|c|c|c|c|}
\hline \multicolumn{8}{|c|}{ Linear weighted Kappa (percentage agreement) } \\
\hline Set of transcripts & Coding pair & Introduction (1) & $\mathrm{OPHI}(2)$ & $\begin{array}{l}\text { Ethnographic } \\
\text { interview (3) }\end{array}$ & $\begin{array}{l}\text { Summary and } \\
\text { Goal-setting (4\&5) }\end{array}$ & $\begin{array}{l}\text { Consultation } \\
\text { and advice (6) }\end{array}$ & Evaluation (7) \\
\hline \multicolumn{8}{|l|}{ Initial piloting } \\
\hline 1 & $1 a$ & $0.79(87)$ & $0.66(68.8)$ & $0.48(56.3)$ & $0.5(58)$ & $\begin{array}{l}\text { 6: } 0.22(35) \\
6(2): 0.44(65)\end{array}$ & $0.37(44)$ \\
\hline 1 & $1 b$ & $0.93(87)$ & $0.40(56.3)$ & $0.2(43.8)$ & $0.23(41.7)$ & $\begin{array}{l}\text { 6: } 0.11(58.8) \\
6 \text { (2): } 0.01(41)\end{array}$ & $0.11(33.3)$ \\
\hline 2 & $1 b$ & $0.65(66.7)$ & $0.27(43.8)$ & $0.57(62.5)$ & $0.28(50)$ & $\begin{array}{l}6: 0.16(47) \\
6(2): 0.13(35.3)\end{array}$ & $0.36(55.6)$ \\
\hline 1 & 2 & $0.40(60)$ & $0.50(62.5)$ & $0.49(56.3)$ & $0.15(37.5)$ & $\begin{array}{l}\text { 6: } 0.5(64.7) \\
6 \text { (2): } 0.08(47)\end{array}$ & $0.16(33.3)$ \\
\hline 2 & 2 & $0.50(53.3)$ & $0.68(75)$ & $0.81(81.3)$ & $0.42(54)$ & $\begin{array}{l}\text { 6: } 0.32(41) \\
6 \text { (2): } 0.34(47)\end{array}$ & $0.54(66)$ \\
\hline \multicolumn{8}{|c|}{ After initial piloting } \\
\hline 3 & 3 & $0.70(80)$ & $0.75(81.3)$ & $0.66(75)$ & $\begin{array}{l}\text { 4: } 0.78(82.6) \\
\text { 5: } 0.37(60.9)\end{array}$ & $0.58(82.4)$ & $0.33(55.6)$ \\
\hline 4 & 3 & $0.71(73.3)$ & $0.87(87.5)$ & $1.00(100)$ & $0.56(59.6)$ & $0.3(58.8)$ & $0.71(87.5)$ \\
\hline 5 & 3 & $0.57(66.7)$ & $0.56(68.8)$ & $0.48(62.5)$ & $0.50(69.6)$ & $0.42(56.3)$ & $0.5(50)$ \\
\hline 5 & 4 & $0.56(60)$ & $0.69(81)$ & $0.67(75)$ & $0.43(65)$ & $0.61(68.8)$ & $0.67(75)$ \\
\hline 5 & 5 & $0.49(66.7)$ & $0.87(87.5)$ & $0.67(75)$ & $0.4(56.5)$ & $0.31(62.5)$ & $0.27(37.5)$ \\
\hline 6 & 4 & $0.67(73.3)^{\mathrm{a}}$ & $0.87(87.5)^{\mathrm{a}}$ & $0.81(87.5)$ & $0.37(52)$ & $0.54(81.3)$ & $1.00(100)$ \\
\hline $8^{1}$ & 4 & $0.7(80)^{\mathrm{a}}$ & $0.94(93.8)^{a}$ & $0.55(68.8)$ & $0.46(60.9)$ & $0.63(75)$ & $0.73(75)$ \\
\hline 12 & 4 & $0.66(80)^{a}$ & $0.81(87.5)^{a}$ & $0.87(87.5)$ & $0.77(88.2)$ & $0.93(93.3)^{a}$ & $0.58(75)$ \\
\hline 1 & 4 & - & - & $0.57(70.6)$ & $0.59(70.6)$ & $0.66(73.3)^{a}$ & $1.00(100)$ \\
\hline 2 & 4 & - & - & $0.81(87.5)^{\mathrm{a}}$ & $0.34(58.8)$ & $0.615(80)^{\mathrm{a}}$ & $0.39(62.5)$ \\
\hline 9 & 4 & - & - & $0.66(75)^{\mathrm{a}}$ & $0.64(76.5)^{a}$ & - & $0.74(75)^{\mathrm{a}}$ \\
\hline 10 & 4 & - & - & $0.81(81)^{\mathrm{a}}$ & $0.76(76.5)^{a}$ & - & $0.73(75)^{\mathrm{a}}$ \\
\hline 11 & 4 & - & - & - & $0.49(70.6)$ & - & $0.77(87.5)^{\mathrm{a}}$ \\
\hline \multirow[t]{2}{*}{3} & 4 & - & - & - & 4: $0.94(94.1)^{\mathrm{a}}$ & - & - \\
\hline & & & & & 5: $0.82(88.2)^{a}$ & & \\
\hline 4 & 4 & - & - & - & $0.44(82.4)^{\mathrm{a} 2}$ & - & - \\
\hline
\end{tabular}

Note: Missing responses were accounted for in the analysis

andicates agreement $>0.61$ was reached | 1Coding guidelines not changed after coding this set | 2Weighted kappa did not reach $>0.61$ however $>70 \%$ agreement achieved five times in a row and > 0.6 kappa 3 times in last five sets Kappa low due to distribution of responses: lots of 'done' responses, despite only three disagreements

-: Agreement had already been reached and no further sessions needed to be coded until the $10 \%$ checks

\section{Procedure}

Occupational therapists were asked to audio-record all intervention sessions provided that 1) consent for audiorecording was obtained and 2) it was practical to do so (e.g. sessions conducted in a home setting but not those involving community activities). Some sessions were not recorded due to some dyads not consenting to audio-recording at the recruitment stage $(n=8)$. Other reasons included: information technology difficulties (e.g. problems with using the recorder or downloading recordings) or dyads declining audio-recording on the day. Routine audio-recording was chosen to overcome the possibility that occupational therapists may self-select which sessions to record [14].
All data were coded for fidelity by the first author. Ten percent of sets were double coded by the fourth author to check that inter-rater agreement was maintained and to assess for coder drift. If inter-rater agreement was not maintained and coder drift occurred, disagreements were discussed to achieve consensus.

\section{Analysis}

Descriptive statistics (mean \%, range \%) were calculated. These were compared across sessions, occupational therapists and sites.

Components were scored according to whether they were: 'done' (score 2), 'done to some extent' (score 1), 


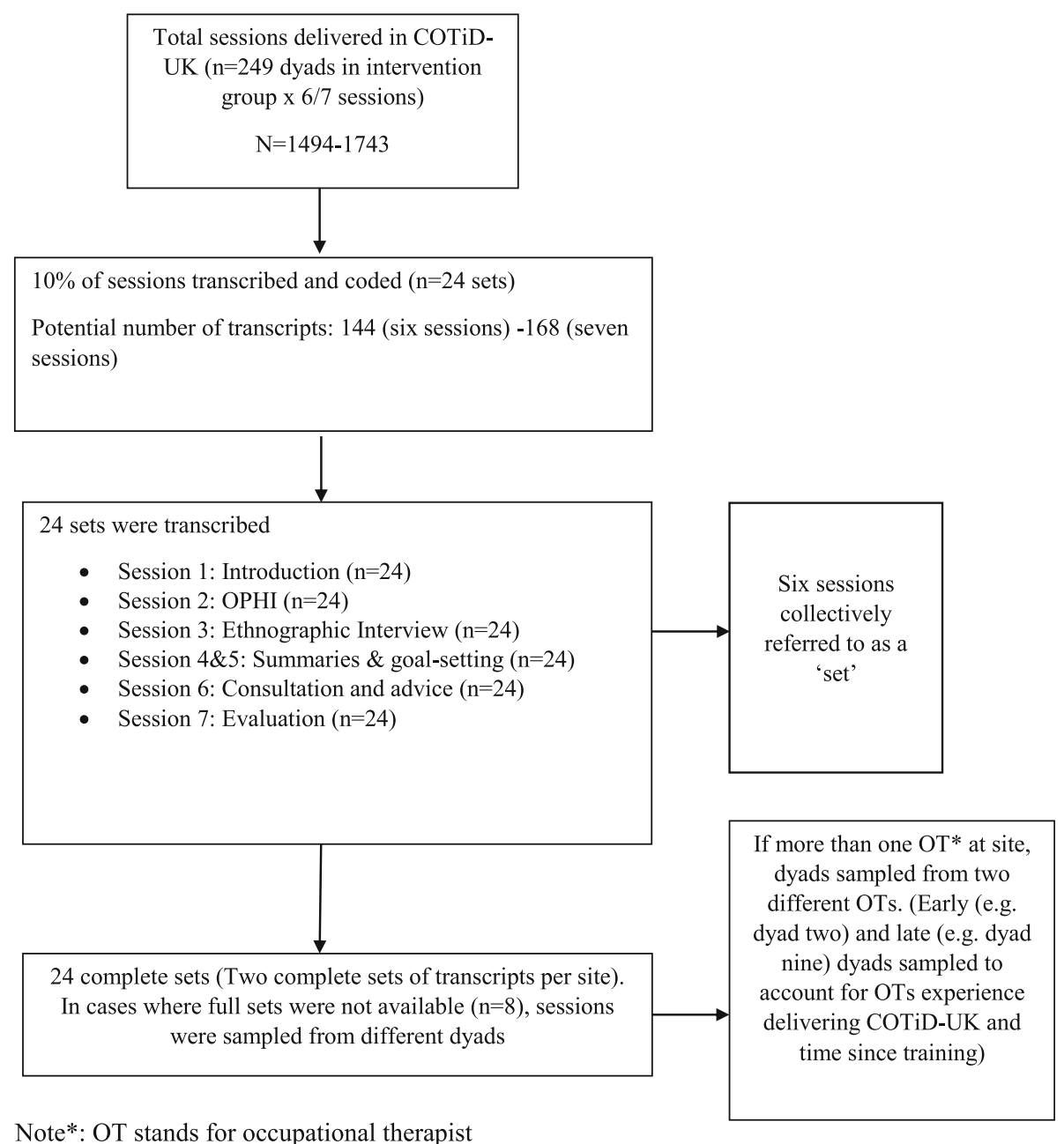

Fig. 2 A flow chart to show the sampling strategy for this study, selected from the COTiD-UK trial

'not done' (score 0), 'delivered in a different session' (coded as '98'), 'not done, not applicable' (coded as '99'). A score of 'done to some extent' was coded when components were neither fully 'done', nor 'not done'. For example, 'done to some extent' was coded if open questions were used to explore two to four topics rather than five or more topics for the OPHI/Ethnographic interviews. 'Not done, not applicable' was coded when the delivery of a component had previously been accounted for, or would be accounted for in the next session (if sessions were delivered together). For example, the component 'assessed the home environment' could be delivered in the first, second or third session. Therefore, when it was coded as 'done' in the first session, this was coded as 'not done, not applicable' in the second and third sessions. To provide a conservative estimate of fidelity and to ensure that fidelity was comparable across occupational therapists and sites, components that were not applicable or delivered in a different session were scored as '0' (not done).
Components that required the absence of behaviour (e.g. 'use of jargon') were reverse coded. A total score and the percentage of the number of components delivered were calculated. A higher score and percentage indicated higher fidelity (80-100\% high fidelity, $51-80 \%$ moderate fidelity and $<50 \%$ low fidelity) [3]. The percentages of sessions for which individual components were 'done', 'done to some extent', 'not done', 'not applicable' or 'delivered in a different session' were calculated.

\section{Results}

\section{Quality of measures}

Out of 2696 completed sessions (the total number of COTiD-UK sessions across all sites), 1409 audio-recordings were made (52.3\%). Twenty-four sets of audio-recordings were chosen for transcription ( $n=$ between 144 and 168 transcripts, depending on whether six or seven sessions were delivered). From these transcripts, 137 transcripts were available and were coded, seven were missing and two 
were labelled incorrectly and could not be coded. Overall, 84 components were scored 'not applicable' (Introduction: 22, OPHI: 32, Ethnographic interview: 24, Summary and Goal-setting (1 and 2): 6 and 3, Consultation and advice: 2, Evaluation: 1), and 27 components were scored 'not done, delivered in a different session' (Introduction: 1, Ethnographic interview: 1, Summary and Goal-setting (1 and 2): 6 and 18, Consultation and advice: 1).

See Table 2 for weighted Kappa and percentage agreement across sessions. Inter-rater agreements for the $10 \%$ of sets that were double coded were all $>.61$, including the Introduction session $(\kappa=0.6-0.8)$, OPHI $(\kappa=0.8-$ 0.9 ), Ethnographic interview ( $\kappa=0.7-0.9)$, and Summary and Goal-setting $(\kappa=0.7-1)$. Agreement for the Consultation and advice session dropped below the required threshold for set eight $(\kappa=0.5)$, but then agreement was reached for sets $13(\kappa=0.8)$ and $16(\kappa=0.8)$. Agreement for the Evaluation session dropped below the required threshold for sets eight $(\kappa=0.5), 13(\kappa=0.3)$ and $14(\kappa=$ $0.3)$, but then agreement was reached for sets $15(\mathrm{~K}=$ $1.0)$ and $16(\kappa=0.9)$. For set 24 , agreement for the Consultation and advice $(\kappa=-0.1)$ and Evaluation sessions $(\kappa=0.2)$ were particularly low. In this set, the Consultation and advice session and Evaluation session were combined and delivered by the occupational therapist in one session and there was no clear distinction as to which components belonged to which session; therefore, it was difficult to code with high agreement.

\section{Measuring fidelity of delivery of the COTID-UK intervention}

Table 3 reports the fidelity of delivery scores across sessions, occupational therapists and sites.

Overall, a mean of $52.4 \%$ (range: $30-86.7 \%$ ) of components were delivered as planned in the Introduction session, $75.5 \%$ (range: $62.5-90.6 \%$ ) in the OPHI session, 71.9\% (range: $56.3-84.4 \%$ ) in the Ethnographic interview, 71.7\% (range: 52.9-94.1\%) in the Summary and Goal- setting session, $65.6 \%$ (range: $30-86.7 \%$ ) in the Consultation and advice session, and 69\% (range: $43.8-87.5 \%$ ) in the Evaluation session. The second Summary and Goalsetting session was delivered with low fidelity (44.1\% of components; range: 29.4-55.9\%). This shows that COTiD-UK was delivered with moderate fidelity overall.

The percentages of components that were delivered ('done', 'done to some extent', 'not done', 'not applicable', or 'delivered in a different session') in each session are reported in Additional file 4. Component numbers in Additional file 4 directly correspond with component numbers in the COTiD-UK checklists. For example, components that were frequently 'not done' for the Summary and Goal-setting session were: 'checked they understood information from family carer' (component five, $n=14,58.3 \%$ not done), 'summarised their own views' (component six, $n=13,54.2 \%$ not done) and 'told participants to start to carry out activities' (component $14, n=13,54.2 \%$ not done). Components that were frequently 'done' for the Summary and Goal-setting session were: 'described what will happen in session' (component one, $n=24,100 \%$ done), 'discussed potential activities' (component seven, $n=24,100 \%$ done), 'set at least one goal' (component eight, $n=22,91.7 \%$ done) and 'prompted the person with dementia and family carer to speak' (component 15, $n=23,95.8 \%$ done).

\section{Comparing fidelity of delivery across intervention sites and OTs}

As Table 3 shows, fidelity of delivery for each session varied across sites (Introduction: 26.7-78.4\%, OPHI: 70.3-82.8\%, Ethnographic interview: $67.2-76.6 \%$, Summary and Goal-setting: 57.4-91.2\%, Consultation and advice: $30-86.7 \%$ and Evaluation: $43.8-78.1 \%$ ) and occupational therapists (Introduction: 26.7-78.4\%, OPHI: 62.5-90.6\%, Ethnographic interview: 56.3-84.4\%, Summary and Goal-setting: 52.9-94.1\%, Consultation and advice: $30-86.7 \%$ and Evaluation: $43.8-81.3 \%)$. This

Table 2 Weighted Kappa and percentage agreement for 10\% of COTiD-UK data that were double coded during the main fidelity assessment

\begin{tabular}{|c|c|c|c|c|c|c|}
\hline \multirow[t]{2}{*}{ Set } & \multicolumn{6}{|c|}{ Weighted Kappa (\%) } \\
\hline & Introduction (1) & $\mathrm{OPHI}(2)$ & $\begin{array}{l}\text { Ethnographic } \\
\text { interview (3) }\end{array}$ & $\begin{array}{l}\text { Summary and } \\
\text { Goal-setting (4\&5) }\end{array}$ & $\begin{array}{l}\text { Consultation } \\
\text { and advice (6) }\end{array}$ & Evaluation (7) \\
\hline $8\left(^{\mathrm{a}}\right)$ & $0.6(66.7)$ & $0.9(93.8)$ & $0.8(81.3)$ & $0.7(82.4)$ & $0.5(73.3)$ & $0.5(75)$ \\
\hline 13 & - & - & - & - & $0.8(86.7)$ & $0.3(62.5)$ \\
\hline 14 & - & - & - & - & - & $0.3(75)$ \\
\hline 15 & - & - & - & - & - & $1(100)$ \\
\hline $16\left(^{\mathrm{a}}\right)$ & $0.8(86.7)$ & $0.8(81.3)$ & $0.7(81.3)$ & $1(100)$ & $0.8(80)$ & $0.9(87.5)$ \\
\hline $24\left({ }^{\mathrm{a}}\right)$ & No transcript & $0.8(81.3)$ & $0.9(87.5)$ & $0.7(82.4)$ & $-0.1(33.3)$ & $0.2(37.5)$ \\
\hline
\end{tabular}

(a) Sets were selected for double coding

-: Agreement had already been reached and no further sessions needed to be coded until the next sampled session

No transcript - refers to sessions where transcripts were not available to code 
Table 3 Fidelity of delivery scores (mean \%, (range \%)) for components delivered across COTiD-UK sessions across sites

\begin{tabular}{|c|c|c|c|c|c|c|c|}
\hline \multirow[t]{3}{*}{ Site } & \multicolumn{7}{|c|}{ Session (Mean \% (Range \%)) } \\
\hline & Introduction (1) & OPHI (2) & $\begin{array}{l}\text { Ethnographic } \\
\text { interview (3) }\end{array}$ & $\begin{array}{l}\text { Summary and } \\
\text { Goal-setting (4\&5) }\end{array}$ & $\begin{array}{l}\text { Consultation } \\
\text { and advice (6) }\end{array}$ & Evaluation (7) & $\begin{array}{l}\text { Summary and } \\
\text { Goal-setting (2) } \\
(4 \& 5(2))^{\mathrm{b}}\end{array}$ \\
\hline & $\begin{array}{l}52.4 \%(26.7-86.7) \text { or } \\
47.5^{\mathrm{a}}(13.3-86.7)\end{array}$ & $75.5(62.5-90.6)$ & $71.9(56.3-84.4)$ & $71.7(52.9-94.1)$ & $65.6(30-86.7)$ & $69.0(43.8-87.5)$ & $44.1(29.4-55.9)$ \\
\hline $\bar{A}$ & $26.7\left(13.3^{\mathrm{a}}-40\right)$ & $76.6(75-78.1)$ & $70.3(62.5-78.1)$ & $57.4(52.9-61.8)$ & $30.0(30.0)$ & $68.8(56.3-81.3)$ & $51.5(47.1-55.9)$ \\
\hline B & $58.3(53.3-63.3)$ & $82.8(78.1-87.5)$ & $76.6(75-78.1)$ & $91.2(88.2-94.1)$ & $86.7(86.7)$ & $71.9(68.8-75.0)$ & - \\
\hline C & $35.0(33.3-36.7)$ & $82.8(75-90.6)$ & $70.3(56.3-84.4)$ & $77.9(73.5-82.4)$ & $55.0(53.3-56.7)$ & $75.0(75)$ & - \\
\hline $\mathrm{D}$ & $55.0(53.3-56.7)$ & $75.0(68.8-81.3)$ & $75.0(68.8-81.3)$ & $64.7(61.8-67.7)$ & $80.0(80.0)$ & $65.6(56.3-75)$ & - \\
\hline E & $28.3(26.7-30)$ & $70.3(62.5-78.1)$ & $75.0(68.8-81.3)$ & $72.1(52.9-91.2)$ & - & $75.0(75)$ & - \\
\hline $\mathrm{F}$ & $28.3\left(13.3^{\mathrm{a}}-43.3\right)$ & $71.9(65.6-78.1)$ & $67.2(62.5-71.9)$ & $77.9(70.6-85.3)$ & $63.3(53.3-73.3)$ & $78.1(75-81.3)$ & - \\
\hline G & $68.3(60-76.7)$ & $73.4(65.6-81.3)$ & $68.8(62.5-75.0)$ & $83.8(76.5-91.2)$ & $71.7(70-73.3)$ & $65.6(56.3-75)$ & - \\
\hline $\mathrm{H}$ & $68.3(56.7-80)$ & 78.1 (75-81.3) & $68.8(68.8)$ & 73.5 (67.7-79.4) & $71.7(66.7-76.7)$ & 65.6 (56.3-75) & - \\
\hline । & $26.7\left(13.3^{\mathrm{a}}-40\right)$ & 73.4 (71.9-75) & $73.4(65.6-81.3)$ & $64.7(64.7)$ & 71.7 (70-73.3) & $43.8(43.8)$ & - \\
\hline J & 36.7 (33.3-40) & $73.4(68.8-78.1)$ & $73.4(65.6-81.3)$ & $63.2(55.9-70.6)$ & - & $65.6(62.5-68.8)$ & - \\
\hline K & $60.0(53.3-66.7)$ & $76.6(68.8-84.4)$ & 71.9 (71.9) & $69.1(58.8-79.4)$ & $46.7(46.7)$ & 78.1 (75-81.3) & - \\
\hline L & $78.4(70-86.7)$ & $71.9(65.6-78.1)$ & $71.9(68.8-75.0)$ & $64.7(64.7)$ & $53.3(53.3)$ & $75.0(62.5-87.5)$ & $29.4(29.4)$ \\
\hline
\end{tabular}

Note: Max fidelity (100\%): Introduction: $n=30$, OPHI and Ethnographic interview: $n=32$, Summary and Goal-setting: $n=34$, Consultation and advice: $n=30$, Evaluation: $n=16$

Components that were N/A are coded as missing and thus scored ' 0 ' in percentage calculations, therefore fidelity for individual sets may be underestimated ${ }^{a}$ Three session 1's were missing - cannot tell fidelity of these. Can only tell if the observations have been carried out, thus leading to some sets having only $13.3 \%$ fidelity. Average without these data points also provided (without ${ }^{a}$ ) $\mid{ }^{b} 4 \& 5(2)=$ second Summary and Goal-setting transcript when OTs have delivered

$4 \& 5$ separately

-: No transcript available

To ensure site anonymity, site numbers have been shuffled up so that numbers 1-15 do not directly correspond to letters A-L

shows that whilst COTiD-UK was delivered with moderate fidelity overall, not all occupational therapists and sites achieved moderate fidelity across all sessions.

\section{Discussion}

\section{Key findings}

In Study 1, a systematic method was used to create a reliable measure of fidelity and apply it to measure fidelity of delivery of the COTiD-UK intervention. Results found that the intervention was delivered moderately well across COTiD-UK sessions. This means that if the COTiD-UK trial finds that the intervention is associated with improved activities of daily living or quality of life, there can be a reasonable degree of confidence that these effects were the result of the planned intervention. If COTiD-UK is found not to be effective, these findings indicate that the intervention was either not effective, or influenced by other factors (e.g. lack of engagement or contamination within the usual care group). As fidelity of COTiD-UK was moderate, and not high, this indicates that some content was not delivered as planned, with variation noted across sites and occupational therapists. Findings may be used to explain the relationship between RCT outcomes and qualitative findings.

\section{Findings in relation to previous research}

In addition to the fidelity of intervention delivery, the psychometric qualities of fidelity measures can inform the interpretation of intervention evaluation effects [15]. The findings from Study 1 are consistent with previous research indicating that it is difficult to achieve good inter-rater reliability when measuring fidelity [26, 27]. Many rounds of coding and amendments to the coding guidelines and checklists were required to reach good agreement. To help achieve agreement, clear definitions of components were developed and provided to make guidelines easier to use and limit subjectivity in responses, as recommended by previous research [10, 26, 28-30]. Furthermore, once agreement was maintained it was necessary to monitor fidelity throughout the fidelity assessment to identify coding drift. This importance of monitoring fidelity throughout the assessment is highlighted in Study 2, as there were instances where agreement dropped below the required threshold. Whilst this was a time-consuming process, monitoring of agreement throughout the fidelity assessment was found to be feasible.

The average fidelity of delivery of intervention components across the intervention sessions ranged from 52.4-75.5\%. This shows that complex occupational therapy interventions for people with dementia, like other behavioural interventions, are not fully delivered as planned [10, 11, 14, 31]. 
Previous research indicates that fidelity often varies across sessions, sites and providers [10, 16]; the results of Study 2 are consistent with these findings. An explanation for differences in fidelity across sessions could lie in the degree of structure and/or provision of materials for some sessions. For example, occupational therapists were given example questions for the OPHI and Ethnographic interviews and were asked to fill in a goal-setting form for the Summary and Goal-setting session. The clear structure of these sessions and the provision of materials may have prompted the delivery of some components, thus increasing fidelity. Following delivery of the goal-setting components, the intervention was then tailored towards the dyads' individual goals, which may have made it more challenging for occupational therapists to deliver the intervention with fidelity.

Differences across occupational therapists and sites could also reflect the difference in occupational therapists' backgrounds and skills. All COTiD-UK providers were occupational therapists and will have shared experience of occupational therapy training. Yet, individual occupational therapists differ in the level of professional experience in working with people with dementia and their family carers, which could have also contributed towards differences in fidelity. Additionally, differences in fidelity may be attributable to different work environments, since COTiD-UK took place across multiple sites. For example, some occupational therapists may have had peers (whom they could seek advice and support from) whereas others may have delivered the intervention with limited peer support. All occupational therapists within COTiD-UK were offered supervision. However, supervision models differed between peer, group and individual supervision, which could have influenced the fidelity of delivery and contributed towards the observed differences.

Differences across occupational therapists and sites could also reflect the levels of tailoring towards individual needs in different sessions. Occupational therapy is a complex dynamic process that comprises multiple practices, the implementation of which is individualised, with the relationship between the person(s) and therapist being fundamental as the process necessitates the active involvement of the person(s) and therapist working in partnership [32]. Hence, occupational therapists may have decided that some components were not relevant in certain situations with particular dyads. Alternatively, occupational therapists may have needed to adapt to a situation that arose on the day (e.g. managing a crisis), which may have influenced fidelity. Although occupational therapists were asked to include explanatory notes within the COTiDUK checklists, the extent to which this was completed varied, and so limited the degree to which this data could be used to inform the fidelity analysis.
This fidelity assessment identified that some aspects of COTiD-UK were predominantly delivered as standalone sessions to all participants, whereas other aspects were delivered more flexibly. Triangulation of findings with the COTiD-UK checklists and supervision records indicated that there were many reasons for this, depending on the session or aspect of the intervention. For example, the OPHI, Ethnographic Interview and Summary and Goal-setting sessions were delivered as planned to all participants. On the other hand, components of the Introduction and Evaluation sessions were sometimes integrated at the beginning or end of other sessions. Also, in practice, the occupational therapist often delivered some of the Introduction components either: by telephone when setting up the appointment; or prior to turning the audio-recorder on; with the latter situation usually influenced by needing to establish rapport and confirm consent. Not all participants received a Consultation and advice session, and in fact 'Consultation and advice' may better be described as a technique rather than a stand-alone session. In practice, Consultation and advice was often integrated within other sessions; or may not have been delivered at all if it did not relate to the goals set. These findings could indicate that the 'OPHI', 'Ethnographic Interview', and 'Summary and Goal-setting' sessions are the core aspects of COTiDUK. Conversely, the 'Introduction', and 'Evaluation' components may be delivered more flexibly - which hinders recording them to assess fidelity, and techniques such as 'Consultation and advice' are often integrated to other sessions, or not delivered at all, depending on the individual dyads' situation and needs.

The results from Study 2 found that fidelity for COTiDUK was lower than COTiD in Germany; the former was based on audio-recorded data, and the latter was based on provider self-report [8]. The use of different methods may explain differences in fidelity, as self-report may lead to biases such as social desirability or difficulties remembering what was delivered [33]. The checklists used to measure fidelity in this study were different than those used in Germany: in COTiD-UK, more specific components were assessed, operationalised as specific activities that occupational therapists could deliver. For example, in this study, OPHI was a key session which contained 16 components (e.g. open questions, use of visual objects). In Germany, 'interviewing the person with OPHI' was included as one component [9]. Without using the same reliable measures across intervention contexts, it is difficult to compare whether interventions were delivered as planned and whether differences in delivery explain possible differences in intervention effects.

Alternatively, a further explanation for differences in delivery across providers and sites, but also any differences in effectiveness between different COTiD trials 
may be attributable to the design of the intervention(s). Researchers have proposed key differences between pragmatic trials (closer to real life situations) and explanatory trials (highly controlled situations) [34, 35]. The Pragmatic-explanatory continuum indicator summary (PRECIS) tool proposes ten aspects on which pragmatic and explanatory trials may differ, including: participant eligibility criteria, flexibility in the intervention and comparison conditions, practitioner expertise for the intervention and comparison conditions, follow up intensity, primary trial outcomes, participant compliance with prescribed intervention, practitioner adherence to study protocol and analysis of primary outcome [35]. COTiD-UK has not been rated against the PRECIS criteria, but would likely score highly on many of these domains, including practitioner adherence to study protocol (as fidelity was closely monitored throughout the COTiD-UK trial), primary trial outcomes, follow-up intensity and analysis of primary outcomes. Other aspects of COTiD-UK may be nearer to the middle of the pragmatic-explanatory continuum [35]. For example: flexibility in intervention conditions (as the intervention was specified but allowed for occupational therapists tailoring the intervention towards individuals' goals and needs) and practitioner expertise (as COTiD-UK was delivered by a number of occupational therapists with differing levels of experience). This may explain variations in the delivery of COTiD-UK across providers and participants. This may also contribute towards understanding of differences between COTiD-UK and previous COTiD trials; if they were implemented differently.

\section{Limitations}

A limitation of this research is that fidelity may have been underestimated in some instances, since some aspects of COTiD-UK were not captured using audiorecording. These included: components of COTiD-UK delivered outside of the home, telephone calls, interactions which occurred after the session had finished and non-verbal interactions.

Furthermore, 'not applicable' components were scored as 'not done' in the analysis. This decision would particularly affect those sessions where 'not applicable' responses were high, including the Introduction Session $(n=22)$, OPHI $(n=32)$ and Ethnographic interview $(n=24)$. There were a large number of 'not applicable' responses for the Introduction session as many Introduction sessions were delivered at the same time as the OPHI; thus rendering components about the next visit 'not applicable'. Furthermore, there were a large number of 'not applicable' responses for the interviews, as 'assessing the home environment' was included on all three checklists. Therefore, it is likely that the fidelity of these sessions, in particular, may be underestimated.

Whilst data on the treatment as usual pathways were collected for the control group in COTiD-UK, fidelity of
COTiD-UK specific components was not measured in the control condition. Therefore, it is not known exactly what the participants in the control groups received. This undermines the ability to draw conclusions from intervention effects [3]. This is particularly important given the differences in control groups in the RCT of COTiD in the Netherlands and Germany, with one control group receiving one occupational therapy visit and the other not receiving any $[6,8]$. If control groups vary on the type of support delivered, this could influence the effectiveness of the intervention.

One limitation is that we have only measured fidelity in this study. Other factors could also affect intervention outcomes. For example, one unknown aspect is the extent to which dyads engaged with the intervention (e.g. their understanding and ability to perform skills or strategies learnt during the intervention, and whether they can put their plans into practice in daily life) [15]; thus, differential engagement cannot be ruled out as a possible factor which may influence COTiD-UK effects [3]. Similarly, given that this trial had many 'explanatory' characteristics, it is possible that implementation in a real-world setting may differ to that of a trial setting. This may be one reason why we observed many 'not applicable' responses in our fidelity assessment.

\section{Implications}

The fidelity checklists developed for this study are specific to this intervention and do not necessarily apply directly to other interventions. However, the method was adapted from measures developed to measure the fidelity of an intervention to improve independence in dementia (PRIDE [19]. As such, the transferability of the method across these studies suggests that it could potentially be applied to develop reliable measures of fidelity for other complex, psychosocial interventions for people with dementia, or interventions more generally.

The study results can also be used to identify problematic components (those which were frequently 'done to some extent' or 'not done') within each session. For example, further training for the Summary and Goal-setting session may need to focus on: 'checking that they understood the information provided by the family carer' (component five), 'summarising own views from observations and assessments' (component six), 'adapting the activity' (component 10), 'providing information about environmental barriers' (component 11), or 'telling participants that they could start to carry out activities to meet the goals' (component 14). Booster training sessions may be needed to enhance fidelity for difficult to deliver components or instances where fidelity is particularly low.

For researchers and policymakers, findings can inform decisions about whether and how COTiD-UK should be implemented on a wider scale. 


\section{Future research}

The checklists produced by this study could be useful for investigating the extent to which COTiD-UK is delivered with fidelity across varying settings and cultures.

Although fidelity was compared across occupational therapists, differences in fidelity were not compared across their experience, gender and age. Further research could investigate these differences.

Larger fidelity studies will enable multilevel modelling to statistically test the differences in fidelity of delivery across providers and sites and their association with intervention effectiveness. This would help to determine which components of COTiD-UK may be most effective and therefore important to deliver.

\section{Conclusion}

A systematic method for measuring fidelity has been developed and used to reliably assess fidelity of delivery of COTiD-UK. COTiD-UK was delivered with moderate fidelity overall, however, its delivery varied across occupational therapists and sites. Findings from this study inform the interpretation of effects reported in the COTiD-UK RCT and qualitative research. Overall, there can be a reasonable degree of confidence that any intervention effects can be attributed to the COTiD-UK intervention. If COTiD-UK is found to not be effective, these findings indicate that COTiD-UK was either not effective or that other factors which were not measured in this study may have influenced effectiveness (e.g. lack of engagement or contamination from usual care). If COTiD-UK is found to be effective, these findings indicate that the intervention content has the potential to support people with dementia and their family carers to maintain independence, engage in meaningful activity and enhance quality of life. Using these findings to understand the delivery of COTiD-UK across sites and occupational therapists may facilitate the interpretation of both RCT and qualitative findings and provide potential explanations for the level of effectiveness of COTiD-UK.

\section{Supplementary information}

Supplementary information accompanies this paper at https://doi.org/10. 1186/s12877-019-1385-7.

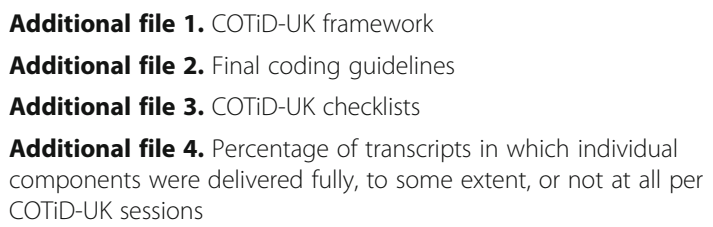

Additional file 4. Percentage of transcripts in which individual components were delivered fully, to some extent, or not at all per COTiD-UK sessions

\section{Abbreviations}

COTiD: Community Occupational Therapy in Dementia; COTiD-

UK: Community Occupational Therapy in Dementia- United Kingdom; NHS: National Health Service; OPHI: Occupational Performance History Interview; PRIDE: Promoting Independence in Dementia; RCT: Randomised
Controlled Trial; SMART: Specific, Measurable, Achievable, Realistic, Timed; UK: United Kingdom

\section{Acknowledgements}

Thank you to Olga Perski, Laura Hull and Harveen Ubhi Kaur for their help with coding. Thank you to Dr. Emma Beard for providing guidance on reliability statistics. Thank you to the Health Psychology Research Group at UCL for commenting on the manuscript.

\section{Authors' contributions}

$\mathrm{MO}$ is the principal investigator for the VALID programme of research. JW is the programme manager for the VALID programme of research. SM, JW, GM and $\mathrm{MO}$ designed the VALID programme of research; which included plans for a fidelity study. HW, with support from SM, AS, IT, JB and JW planned and led the fidelity study. JB organised and managed data collection of audiorecordings. HW developed the COTiD-UK framework and fidelity checklists with feedback from IT, JB, JW, AS and SM. JB HW, HG, TS and JB piloted fidelity coding until reliability was achieved. HW coded all data and HG coded $10 \%$ of data. HW analysed the data with input from all authors. HW drafted the fidelity paper and all authors commented on, and approved the final draft.

\section{Authors' information \\ Not applicable}

\section{Funding}

This study is funded by the National Institute for Health Research (NIHR) [Programme Grants for Applied Research (Grant Reference Number RP-PG0610-10108)]. The views expressed are those of the authors and not necessarily those of the NIHR or the Department of Health and Social Care. The VALID research team acknowledges the support of the National Institute of Health Research Clinical Research Network. This research is sponsored by North East London NHS Foundation Trust (NELFT).

Holly Walton's PhD research is funded by the UCL Economic and Social Research Council Doctoral Training Centre (Grant Reference: ES/J500185/1). Ildiko Tombor's post is funded by a programme grant from Cancer Research UK (CRUK; C1417/A22962). The funding bodies played no role in the design of the study and collection, analysis, and interpretation of data and in writing the manuscript.

\section{Availability of data and materials}

The datasets generated and/or analysed during the current study are not publicly available due to participant confidentiality but are available from the corresponding author on reasonable request.

\section{Ethics approval and consent to participate}

Ethical approval for this study was obtained from: NRES Committee London Camberwell St Giles, REC Reference number: 14/LO/0736. Participants provided informed consent for audio-recording of intervention sessions as part of their written consent to take part in the COTiD-UK trial (see [17] for details of the trial consent process).

\section{Consent for publication}

Not applicable.

\section{Competing interests}

The authors declare that they have no competing interests.

\footnotetext{
Author details

${ }^{1}$ Department of Applied Health Research, University College London, 1-19 Torrington Place, London, UK. ²Department of Behavioural Science and Health, University College London, 1-19 Torrington Place, London, UK. ${ }^{3}$ Research and Development Department, Goodmayes Hospital, North East London NHS Foundation Trust, Essex, UK. ${ }^{4}$ Department of Clinical, Educational and Health Psychology, University College London, 1-19 Torrington Place, London WC1E 7HB, UK. ${ }^{5}$ East Hertfordshire and Broxbourne Adult Disability Team, Hertfordshire County Council, Stevenage, UK. ${ }^{6}$ Division of Psychiatry, Faculty of Brain Sciences, University College London, London, UK. ' Institute of Mental Health, University of Nottingham, Nottingham, UK. ${ }^{8}$ School of Health and Related Research, The University of Sheffield, Sheffield, UK.
} 
Received: 14 September 2018 Accepted: 12 December 2019 Published online: 23 December 2019

\section{References}

1. Alzheimer's Society. Facts for the Media. https://www.alzheimers.org.uk/info/2002 7/news_and_media/541/facts_for_the_media (2017). Accessed 10 Jan 2018.

2. Bellg AJ, Borrelli B, Resnick B, Hecht J, Minicucci DS, Ory M, et al. Enhancing treatment fidelity in health behavior change studies: best practices and recommendations from the $\mathrm{NIH}$ behavior change Consortium. Health Psychol. 2004;23(5):443. https://doi.org/10.1037/0278-6133.23.5.443.

3. Borrelli B. The assessment, monitoring, and enhancement of treatment fidelity in public health clinical trials. J Public Health Dent. 2011;71(s1):S5263. https://doi.org/10.1111/j.1752-7325.2011.00233x.

4. Moncher FJ, Prinz RJ. Treatment fidelity in outcome studies. Clin Psychol Rev. 1991;11(3):247-66. https://doi.org/10.1016/0272-7358(91)90103-2.

5. Vernooij-Dassen M, Moniz-Cook E. Raising the standard of applied dementia care research: addressing the implementation error. Aging Ment Health. 2014;18(7):809-14. https://doi.org/10.1080/13607863.2014.899977.

6. Graff MJL, Vernooij-Dassen MJM, Thijssen M, Dekker J, Hoefnagels WHL, Rikkert MGMO. Community based occupational therapy for patients with dementia and their care givers: randomised controlled trial. BMJ. 2006; 333(7580):1196-9. https://doi.org/10.1136/bmj.39001.688843.BE.

7. Graff MJ, Adang EM, Vernooij-Dassen MJ, Dekker J, Jönsson L, Thijssen M, Rikkert MGO. Community occupational therapy for older patients with dementia and their care givers: cost effectiveness study. BMJ. 2008; 336(7636):134-8. https://doi.org/10.1136/bmj.39408.481898.BE.

8. Voigt-Radloff S, Graff M, Leonhart R, Hüll M, Rikkert MO, Vernooij-Dassen M. Why did an effective Dutch complex psycho-social intervention for people with dementia not work in the German healthcare context? Lessons learnt from a process evaluation alongside a multicentre RCT. BMJ Open. 2011; 1(1):e000094. https://doi.org/10.1136/bmjopen-2011- 000094.

9. Voigt-Radloff S, Graff M, Leonhart R, Schornstein K, Jessen F, Bohlken J, Eschweiler G. A multicentre RCT on community occupational therapy in Alzheimer's disease: 10 sessions are not better than one consultation. BMJ Open. 2011;1(1):e000096. https://doi.org/10.1136/bmjopen-2011- 000096.

10. Lorencatto F, West R, Bruguera C, Michie S. A method for assessing fidelity of delivery of telephone behavioral support for smoking cessation. J Consult Clin Psychol. 2014:82(3):482-91. https://doi.org/10.1037/a0035149.

11. Toomey E, Currie-Murphy L, Matthews J, Hurley DA. Implementation fidelity of physiotherapist-delivered group education and exercise interventions to promote self-management in people with osteoarthritis and chronic low back pain: a rapid review part II. Man Ther. 2015;20(2):287-94. https://doi. org/10.1016/j.math.2014.10.012.

12. Gresham FM, Gansle KA, Noell GH. Treatment integrity in applied behaviour analysis with children. J Appl Behav Anal. 1993;26:257-63. https://doi.org/10. 1901/jaba.1993.26-257.

13. Miller WR, Rollnick S. The effectiveness and ineffectiveness of complex behavioral interventions: impact of treatment fidelity. Contemp Clin Trials. 2014;37(2):234-41. https://doi.org/10.1016/j.cct.2014.01.005.

14. Lorencatto F, West R, Christopherson C, Michie S. Assessing fidelity of delivery of smoking cessation behavioural support in practice. Implement Sci. 2013:8(40). https://doi.org/10.1186/1748-5908-8-40.

15. Walton $\mathrm{H}$, Spector $\mathrm{A}$, Tombor I, Michie S. Measures of fidelity of delivery of, and engagement with, complex, face-to-face health behaviour change interventions: a systematic review of measure quality. Br J Health Psychol. 2017;22(4):872-903. https://doi.org/10.1111/bjhp.12260.

16. Durlak JA. Why program implementation is important. J Prev Interv Community. 1998;17(2):5-18. https://doi.org/10.1300/J005v17n02_02.

17. Wenborn J, Hynes S, Moniz-Cook E, Mountain G, Poland F, Orrell M. Community occupational therapy for people with dementia and family carers (COTiD-UK) versus treatment as usual (valuing active life in dementia [VALID] programme): study protocol for a randomised controlled trial. Trials. 2016;16(65):1-10. https://doi.org/10.1186/s13063-015-1150-y.

18. Kielhofner G, Mallinson T, Crawford C, Nowak M, Rigby M, Henry A, Walens D. Occupational performance history interview II (OPHI-II) version 2.1. Chicago: Model of Human Occupation Clearinghouse, Department of Occupational Therapy, College of Applied Health Sciences, University of Illinois at Chicago; 2004

19. Walton H, Spector A, Williamson M, Tombor I, Michie S. Developing quality fidelity and engagement measures for complex health interventions. $\mathrm{Br} J$ Health Psychol. 2019. https://doi.org/10.1111/bjhp.12394.
20. Creek J. Occupational therapy defined as a complex intervention. Coll Occup Ther Lond. 2003.

21. Lombard M, Snyder-Duch J, Bracken CC. Content analysis in mass communication: assessment and reporting of intercoder reliability. Hum Commun Res. 2002;28(4):587-604. https://doi.org/10.1111/j.1468-2958.2002. tb00826.x.

22. Cohen J. A coefficient of agreement for nominal scales. Educ Psychol Meas. 1960;20(1):37-46.

23. Gwet KL. Handbook of inter-rater reliability. 4th ed. Advanced Analytics: Gaithersburg; 2014.

24. Landis JR, Koch GG. The measurement of observer agreement for categorical data. Biometrics. 1977;33(1):159-74. https://doi.org/10.2307/ 2529310.

25. Feinstein AR, Cicchetti DV. High agreement but low kappa: I. the problems of two paradoxes. J Clin Epidemiol. 1990;43(6):543-9. https://doi.org/10. 1016/0895-4356(90)90158-L.

26. Harting J, van Assema P, van der Molen HT, Ambergen T, de Vries NK. Quality assessment of health counseling: performance of health advisors in cardiovascular prevention. Patient Educ Couns. 2004;54(1):107-18. https:// doi.org/10.1016/S0738-3991(03) 00194-0.

27. Thyrian JR, Freyer-Adam J, Hannover W, Roske K, Mentzel F, Kufeld C, Hapke U. Population-based smoking cessation in women post partum: adherence to motivational interviewing in relation to client characteristics and behavioural outcomes. Midwifery. 2010;26:202-10. https://doi.org/10.1016/j. midw.2008.04.004

28. French SD, Green SE, Francis JJ, Buchbinder R, O'Connor DA, Grimshaw JM, Michie S. Evaluation of the fidelity of an interactive face-to-face educational intervention to improve general practitioner management of back pain. BMJ Open. 2015;5(7):e007886. https://doi.org/10.1136/bmjopen-2015007886.

29. Hardeman W, Michie S, Fanshawe T, Prevost AT, Mcloughlin K, Kinmonth AL. Fidelity of delivery of a physical activity intervention: predictors and consequences. Psychol Health. 2008;23(1):11-24. https://doi.org/10.1080/ 08870440701615948

30. Keith R, Hopp F, Subramanian U, Wiitala W, Lowery J. Fidelity of implementation: development and testing of a measure. Implement Sci. 2010:5(1):99. https://doi.org/10.1186/1748-5908-5-99.

31. Bond GR, Drake RE, McHugo GJ, Rapp CA, Whitley R. Strategies for improving fidelity in the national evidence-based practices project. Res Soc Work Pract. 2009;19(5):569-81. https://doi.org/10.1177/1049731509335531.

32. Pentland D, Kantartzis S, Clausen MG, Witemyre K. Occupational therapy and complexity: defining and describing practice. London: Royal College of Occupational Therapists; 2018. Available from: https://rcot.co.uk

33. Schinckus L, Van den Broucke S, Housiaux M, Consortium DL. Assessment of implementation fidelity in diabetes self-management education programs: a systematic review. Patient Educ Couns. 2014;96(1):13-21. https://doi.org/10. 1016/j.pec.2014.04.002

34. Schwartz D, Lellouch J. Explanatory and pragmatic attitudes in therapeutical trials. J Chronic Dis. 1967;20(8):637-48. https://doi.org/10.1016/00219681(67)90041-0.

35. Thorpe KE, Zwarenstein M, Oxman AD, Treweek S, Furberg CD, Altman DG, Tunis S, Bergel E, Harvey I, Magid DJ, Chalkidou K. A pragmatic-explanatory continuum indicator summary (PRECIS): a tool to help trial designers. J Clin Epidemiol. 2009;62(5):464-75. https://doi.org/10.1016/j.jclinepi.2008.12.011.

\section{Publisher's Note}

Springer Nature remains neutral with regard to jurisdictional claims in published maps and institutional affiliations. 